IMPACTO DEL PROGRAMA PENSIÓN PARA ADULTOS MAYORES (PPAM) EN TABASCO, MÉXICO. PERÍODO 2013-2015

\title{
IMPACTO DEL PROGRAMA PENSIÓN PARA ADULTOS MAYORES (PPAM) EN TABASCO, MÉXICO. PERÍODO 2013-2015
}

José Félix García Rodríguez*, Carlos M. Lezama León”*, Aída Beatriz Armenta Ramírez***, Luis Arturo

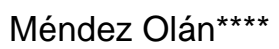

*Doctor en Finanzas Públicas. Profesor Investigador de la Universidad Juárez Autónoma de Tabasco, División Académica de Ciencias Económico Administrativas.

**Maestro en Administración. Subdelegado de Desarrollo Social y Humano. Secretaría de Desarrollo Social de Tabasco.

***Doctora en Economía Regional. Universidad Juárez Autónoma de Tabasco. División Académica de Ciencias Económico Administrativas.

****Maestro en Administración. Universidad Juárez Autónoma de Tabasco. División Académica de Ciencias Económico Administrativas.

Dirección para recibir correspondencia: jfgr55@hotmail.com 
IMPACTO DEL PROGRAMA PENSIÓN PARA ADULTOS MAYORES (PPAM) EN TABASCO, MÉXICO. PERÍODO 2013-2015

\section{RESUMEN}

Objetivo: Analizar el impacto en Tabasco del Programa Pensión para Adultos Mayores (PPAM) entre sus beneficiarios en pobreza extrema, durante el período 2013-2015, buscando confirmar la hipótesis de que dichos beneficiarios mejoraron sus condiciones de bienestar debido a su incorporación al PPAM.

Material y método: Se desarrolló una investigación cuantitativa de tipo descriptivo no experimental. La población objeto de estudio fueron los hogares con adultos mayores en pobreza extrema del municipio de Centro, determinándose un universo poblacional de 4,122 beneficiarios y un marco muestral de 314 hogares identificados en pobreza extrema, obteniéndose por muestreo aleatorio simple una muestra representativa de 173 hogares.

Resultados: Los resultados de la investigación permiten afirmar que los hogares con adultos mayores de 65 años en pobreza extrema mejoraron en general sus condiciones socioeconómicas.

Conclusiones: Los adultos mayores del municipio de Centro, Tabasco, que en el año 2013 se encontraban en condiciones de pobreza extrema, al año 2015 observaron una importante disminución en sus carencias básicas, debido a su incorporación al PPAM.

Palabras clave: Adultos mayores. Bienestar social. Vulnerabilidad. Pobreza. Desigualdad. 
IMPACTO DEL PROGRAMA PENSIÓN PARA ADULTOS MAYORES (PPAM) EN TABASCO, MÉXICO. PERÍODO 2013-2015

\section{ABSTRACT}

Objective: The purpose of this research is to analyze the impact of the Pension Program for Elderly (PPAM) among its beneficiaries in extreme poverty during the period 2013-2015, in order to confirm the hypothesis that these beneficiaries improved their welfare conditions due to their incorporation into PPAM.

Material and method: A non-experimental descriptive quantitative investigation was developed. The study population was the households with elderly people in extreme poverty of the municipality of Centro, determining a population of 4,122 beneficiaries and a sampling frame of 314 households identified in extreme poverty, obtaining by simple random sampling a representative sample of 173 households.

Results: The results of the research allow to affirm that the households with adults over 65 in extreme poverty generally improved their socioeconomic conditions.

Conclusions: Older adults in the municipality of Centro, Tabasco, who were in extreme poverty in the year 2013, observed a significant decrease in their basic deficits in 2015, due to their incorporation into the PPAM.

Keywords: Older adults. Social welfare. Vulnerability. Poverty. Inequality. 
IMPACTO DEL PROGRAMA PENSIÓN PARA ADULTOS MAYORES (PPAM) EN TABASCO, MÉXICO. PERÍODO 2013-2015

\section{INTRODUCCIÓN}

El fenómeno del envejecimiento poblacional cobra cada vez mayor relevancia a nivel internacional y México no es la excepción. En nuestro país, los adultos mayores de hoy día nacieron en una época en la que la mayor parte de la población vivía en condiciones de pobreza. De acuerdo con Székely (2005), en 1950 el 88\% de la población se encontraba en pobreza patrimonial, $73 \%$ en pobreza de capacidades y $61 \%$ en pobreza alimentaria. Además, enfrentaron insuficiencia en provisión de servicios educativos y de salud. Ello dio como resultado varias generaciones con alto grado de analfabetismo, bajos niveles de salud y alta incidencia de desnutrición.

De esta manera, el envejecimiento tiene múltiples facetas que exigen su análisis y demandan su atención. Normalmente, los adultos mayores enfrentan entre otras privaciones problemas de salud, educación, desempleo, baja cobertura en pensiones y protección social, lo que conlleva a problemas de vulnerabilidad, pobreza y desigualdad.

\section{Vulnerabilidad, pobreza y desigualdad}

Para efectos de la investigación, es importante entender las diferencias y similitudes existentes entre los procesos de vulnerabilidad, pobreza y desigualdad que enfrentan los adultos mayores. El Informe de Desarrollo Mundial del Banco Mundial (2001), asocia la vulnerabilidad con la probabilidad de estar expuesto a una gran variedad de riesgos (violencia, crimen, desastres naturales, abandono escolar).

Diversos grupos sociales son vulnerables por que poseen una desventaja por limitaciones físicas, de edad, género, etnia o pobreza. Más allá de las condiciones de pobreza, pobreza extrema y marginación, existen un sinfín de situaciones que comprometen severamente el desarrollo físico e intelectual de las personas y las hacen vulnerables. Entre ellas se encuentran el abandono, la orfandad, la desprotección o desamparo, la indigencia, los diferentes tipos de discapacidades.

En este sentido, el concepto de vulnerabilidad se refiere a la diversidad de situaciones que pueden sucederle a un individuo o grupo social, y al proceso por el cual se está en riesgo. Vulnerabilidad no es exactamente lo mismo que pobreza, sin embargo esta última la incluye. La pobreza hace referencia a una situación de carencia efectiva y actual, mientras que la vulnerabilidad trasciende esta condición, proyectando a futuro la posibilidad de padecerla a 
IMPACTO DEL PROGRAMA PENSIÓN PARA ADULTOS MAYORES (PPAM) EN TABASCO, MÉXICO. PERÍODO 2013-2015

partir de ciertas debilidades que se constatan en el presente. De esta manera, si bien no todos los grupos vulnerables son pobres, todos los pobres son vulnerables.

De acuerdo a la metodología oficial de medición de pobreza multidimensional aplicada por el Consejo Nacional de Evaluación de la Política Social (CONEVAL,2015), pobres son las personas y los hogares cuyos ingresos son insuficientes para adquirir los bienes y servicios que se requieren para satisfacer sus necesidades, y que presentan carencias en al menos uno de los siguientes seis indicadores: rezago educativo, acceso a los servicios de salud, acceso a la seguridad social, calidad y espacios de la vivienda, servicios básicos en la vivienda y acceso a la alimentación.

Asimismo, se consideran en situación de pobreza extrema cuando tienen tres o más de las carencias antes mencionadas, y que además se encuentran por debajo de la línea de bienestar mínimo. Las personas en esta situación disponen de un ingreso tan bajo que aún si lo dedicasen por completo a la adquisición de alimentos, no podrían adquirir los nutrientes necesarios para tener una vida sana, por lo que eliminar la pobreza extrema representa todo un reto institucional, ante lo cual es necesario desarrollar esfuerzos conjuntos entre el Estado y la sociedad en general.

Por su parte, la desigualdad, al igual que la vulnerabilidad, está presente no solo entre los pobres, sin embargo, todos los pobres viven en condiciones de desigualdad. No debe confundirse pobreza con desigualdad, la pobreza se refiere a la situación económica, en tanto que la desigualdad es social y política. La pobreza como concepto hace referencia a la carencia de una persona o población por no poder cubrir sus necesidades básicas; en cambio, la desigualdad es la imposibilidad de acceder a determinados recursos, derechos, obligaciones, beneficios etc. De esta manera, la desigualdad es una manifestación de la pobreza. La desigualdad puede ser social y económica, de género, racial, cultural y geográfica. La brecha entre los más ricos y los más pobres de un determinado lugar permite establecer el nivel de desigualdad de un país o sociedad, y esto acentúa los niveles de pobreza.

Piketty (2014) plantea que la desigualdad, entendida como la forma en que se encuentra distribuida la riqueza entre la sociedad, constituye en la actualidad uno de los temas más debatidos y controversiales dentro de la economía política contemporánea, y que los debates acerca del tema están más cargados de especulaciones teóricas y prejuicios políticos y sociales 
IMPACTO DEL PROGRAMA PENSIÓN PARA ADULTOS MAYORES (PPAM) EN TABASCO, MÉXICO. PERÍODO 2013-2015

que de información y hechos objetivos. La pobreza y la desigualdad son dos conceptos interrelacionados, tanto que para algunos expertos el problema no es la pobreza sino la desigualdad. Otros consideran que ambos deben ser tratados para lograr tener una sociedad justa y equilibrada, ya que no alcanza con el crecimiento económico, sino que además hay que implementar acciones de distribución de la riqueza e inversión social para lograr un desarrollo humano extendido. Al respecto, (García, 2015, p. 31) menciona que "es imprescindible que haya crecimiento económico, estabilidad, competitividad, eficiencia económica junto con desarrollo social; no son aspectos antitéticos, al contrario. La experiencia de los países exitosos indica que el desarrollo social es un poderoso motor del crecimiento económico".

En México, el bajo nivel de ingreso y las bajas tasas de ahorro han reducido a la mayor parte de los adultos mayores a una situación de pobreza, condición que se agravará en los próximos años debido a la transformación de la pirámide poblacional, lo que implica una mayor cantidad de adultos mayores respecto a la población total. En consecuencia, resulta de suma importancia elaborar políticas públicas adecuadas que atiendan de manera efectiva a este grupo de población tanto en zonas urbanas como rurales. La condición de vulnerabilidad de los adultos mayores no solo afecta a este grupo etario, sino también a los hogares en los que residen. El hecho de que los hogares en condiciones de pobreza no cuenten con los recursos necesarios para enfrentar gastos en situaciones catastróficas de salud, da lugar a que tengan que deshacerse de sus bienes y patrimonio, teniendo una disminución irrecuperable de sus activos ante gastos imprevistos, aumentando así la posibilidad de caer en condiciones de pobreza o de perpetuar la transmisión intergeneracional de la misma.

En este contexto, y con el propósito de mejorar la calidad de vida de los adultos mayores, en México el Gobierno Federal a través de la Secretaría de Desarrollo Social (SEDESOL), puso en marcha el Programa Pensión para Adultos Mayores (PPAM), el cual atiende a las personas adultas mayores de 65 años en adelante, con cobertura nacional. Los beneficiarios del mismo reciben apoyos económicos de $\$ 580$ pesos mexicanos mensuales pagaderos de forma bimestral. El propósito del PPAM es contribuir a disminuir las condiciones de pobreza y vulnerabilidad de los Adultos Mayores, tomando en cuenta su vulnerabilidad por seguridad social y por ingresos. Esta nueva estrategia para la seguridad social amplió la cobertura del programa al aumentar el rango a una edad mínima de 65 años para los adultos mayores que no reciban ingresos por concepto de pensión o jubilación de tipo contributivo. De esta manera, el programa busca asegurar un ingreso mínimo y apoyos de protección social a las personas 
IMPACTO DEL PROGRAMA PENSIÓN PARA ADULTOS MAYORES (PPAM) EN TABASCO, MÉXICO. PERÍODO 2013-2015

adultas mayores de 65 años de edad que no reciben un ingreso mensual superior a $\$ 1,092$ pesos por concepto de jubilación o pensión de tipo contributivo.

El PPAM ha demostrado que la combinación de la entrega de una pensión monetaria de tipo no contributivo, más la realización de acciones para aminorar el deterioro de la salud física y mental a través de la creación de una red social en favor de este grupo social, aunados a acciones de protección social coordinadas entre todo el sector público, mejoran efectivamente las condiciones de vida de las personas adultas mayores.

\section{MATERIAL Y MÉTODO}

El objetivo de la investigación es evaluar el impacto que tuvo el Programa Pensión para Adultos Mayores en los hogares beneficiarios en extrema pobreza del municipio de Centro, Tabasco, durante el período 2013-2015. Específicamente, se buscó dar respuesta a las siguientes preguntas de investigación:

a) ¿Cuál es la situación de bienestar económico y social de los hogares beneficiarios del Programa Pensión para Adultos Mayores?

b) ¿Qué carencias sociales se han logrado disminuir en los hogares investigados a partir de la aplicación del Programa?,

c) ¿Cuántos de los hogares beneficiarios del Programa aún se encuentran en pobreza extrema?

En congruencia con lo anterior, la hipótesis de investigación fue: los hogares beneficiarios del Programa Pensión para Adultos Mayores en el municipio de Centro, Tabasco que se encontraban en pobreza extrema en el año 2013, lograron mejorar sus condiciones socioeconómicas en el año 2015.

\section{Enfoque, tipo y diseño de la Investigación}

Se llevó a cabo una investigación con enfoque cuantitativo, de tipo descriptivo no experimental, a partir de un universo poblacional y la aplicación de un proceso de muestreo aleatorio simple. Al respecto, Hernández, Fernández y Baptista (2006, p. 5) expresan que "el enfoque cuantitativo usa la recolección de datos para probar hipótesis, con base en la medición numérica y el análisis estadístico, para establecer patrones de comportamiento y probar teorías". 
IMPACTO DEL PROGRAMA PENSIÓN PARA ADULTOS MAYORES (PPAM) EN TABASCO, MÉXICO. PERÍODO 2013-2015

La población objeto de estudio se definió como los hogares con adultos mayores en pobreza extrema del municipio de Centro, Tabasco, que fueron incorporados en el año 2013 al PPAM y que al momento de inscribirse al programa tuvieran una edad entre 65 y 70 años. De esta manera, se determinó un universo poblacional de 4,122 beneficiarios. Asimismo, se establecieron como marco muestral un total de 314 hogares que fueron identificados en pobreza extrema.

El tamaño adecuado de la muestra representativa de la población objeto de estudio es de 173 hogares, y se determinó a partir de los siguientes criterios: 1) Personas en condición de pobreza extrema; 2) nivel deseado de confianza, y 3) margen de error aceptable. Dicho tamaño de muestra se calculó a través de la aplicación de la siguiente fórmula:

$n=\frac{N z^{2} p q}{e^{2(N-1)+z p q}}$

Donde:

$\mathrm{n}=$ Tamaño de la muestra.

$\mathrm{N}=$ Tamaño de la población.

Z= Nivel de confianza del 95\%; [1.96]

$\mathrm{p}=$ Probabilidad de éxito es de un 50\% (0.50)

$q=$ Probabilidad de fracaso $100 \%-50 \%=(0.50)$

e= Precisión (Error máximo admisible en términos de proporción) 5\%

Como instrumento de recolección de la información de campo se aplicó un cuestionario adaptado a las necesidades de la investigación, adaptado a partir del "Cuestionario único de Información Socioeconómica (CUIS)", instrumento usado para la evaluación de criterios de identificación de beneficiarios de los programas sociales que tiene a su cargo la Secretaría de Desarrollo Social (SEDESOL). Dicho instrumento es aplicado para dar cumplimiento a los "Lineamientos y Criterios Generales para la definición, identificación y medición de la pobreza" del Consejo Nacional de Evaluación de la Política de Desarrollo Social (CONEVAL, 2015), publicados en el Diario Oficial de la Federación el 16 de junio de 2010. Asimismo, el CUIS forma parte integral de las Reglas de Operación del Programa Pensión para Adultos Mayores, para el ejercicio fiscal 2015, las cuales fueron publicadas en el Diario Oficial de la Federación el sábado 27 de diciembre del 2014, en su octava sección. 
IMPACTO DEL PROGRAMA PENSIÓN PARA ADULTOS MAYORES (PPAM) EN TABASCO, MÉXICO. PERÍODO 2013-2015

El cuestionario aplicado en la investigación se centra en la información relacionada con los indicadores de ingreso corriente per cápita, rezago educativo promedio en el hogar, acceso a los servicios de salud, acceso a la seguridad social, calidad y espacios de la vivienda, acceso a los servicios básicos en la vivienda, y acceso a la alimentación. Consta de 39 preguntas, de las cuales 34 se enfocan al ingreso y seis tipos de carencias, de conformidad con los criterios que da el CONEVAL para conocer la situación socioeconómica en la que se encuentran las personas. Las restantes cinco preguntas tienen que ver con condiciones socioeconómicas, en cuanto a calidad de espacios en la vivienda y acceso a la alimentación.

\section{RESULTADOS}

En lo que corresponde a rezago educativo promedio en el hogar, se observó una disminución de casi un $11 \%$ durante el período de análisis. De todas las carencias sociales analizadas, ésta es la que tuvo un menor decremento. Sin embargo, constituye un avance sustancial por tratarse de adultos mayores, grupo poblacional vulnerable donde son mayores los esfuerzos y menores los resultados alcanzados en materia educativa (ver tabla 1).

Los resultados de la aplicación del PPAM son más evidentes en la dimensión de acceso a los servicios de salud, observándose una importante disminución en su incidencia. Así, del 100\% de hogares que enfrentaban dicha carencia en el año 2013, al 2015 sólo el 5.5\% continuaban sin acceso a dichos servicios. Asimismo, se observó que de las 68 personas que lograron superar esta carencia, el 97\% lo hizo gracias a su incorporación al programa sanitario federal conocido como Seguro Popular de Salud, en tanto que el 3\% restante fueron inscritos al Instituto Mexicano del Seguro Social. Este resultado permite visualizar que la política actual del gobierno federal en materia sanitaria ha contribuido a mejorar la calidad de vida de la población adulta mayor (ver tabla 1).

En lo que corresponde al indicador de acceso a la seguridad social, la investigación arroja también un avance sustancial, puesto que se logró erradicar por completo dicha carencia, observándose que de los 173 adultos mayores que en 2013 se encontraban con esta carencia, al 2015 todos habían logrado superar este rezago. Debe aclararse que lo anterior se logró gracias a la incorporación de todos los adultos mayores al PPAM. Otro hecho relevante de la investigación es el hallazgo de que el 30\% de los hogares entrevistados perciben hasta dos pensiones por parte del PPAM. Ello debido a la presencia de dos adultos mayores en un mismo hogar, tendencia demográfica irreversible en nuestro país (ver tabla 1). 
IMPACTO DEL PROGRAMA PENSIÓN PARA ADULTOS MAYORES (PPAM) EN TABASCO, MÉXICO. PERÍODO 2013-2015

La dimensión calidad y espacios de la vivienda es la única carencia social que se incrementó, pues durante el período de análisis el número de hogares con esta carencia se elevó un 11.44\%. En contraste, la dimensión acceso a los servicios básicos en la vivienda observó una disminución sustancial del 69.34 \%. De esta manera, durante el período de análisis, 95 de 137 hogares con adultos mayores lograron salir de dicha carencia. Respecto a la variable acceso a la alimentación, el análisis arroja un importante avance en materia de seguridad alimentaria durante el período analizado, ya que de 164 hogares con adultos mayores que vivían con carencia alimentaria, al 2015 únicamente 9 hogares continuaban en dicha condición. Esto representa una disminución del $94.51 \%$ en esta carencia (ver tabla 1).

Un resultado contrastante corresponde a la carencia por ingreso corriente per cápita, pues aunque se observó un avance positivo, éste es marginal, toda vez que la carencia de nivel de ingreso por debajo de la línea de bienestar se redujo apenas un poco menos del 2\%, y el ingreso por debajo de la línea de bienestar mínimo un poco menos del 3\%. Al respecto, cabe mencionar que este resultado es congruente, toda vez que el PPAM lo que busca es elevar el bienestar general de sus beneficiarios a través de asegurarles un ingreso mínimo acompañado con otros apoyos de protección social (ver tabla 1).

En conclusión, puede decirse que durante el período de estudio, las carencias sociales de los hogares con adultos mayores beneficiados con el PPAM observaron una importante disminución, exceptuando el indicador de calidad y espacios en la vivienda. En lo que corresponde al ingreso corriente per cápita de los hogares encuestados, solo se observó un avance marginal, lo que constituye un aspecto en el cual deberán incidir los esfuerzos institucionales orientados a mejor las condiciones socioeconómicas de los adultos mayores del municipio de Centro, Tabasco. 
IMPACTO DEL PROGRAMA PENSIÓN PARA ADULTOS MAYORES (PPAM) EN TABASCO, MÉXICO. PERÍODO 2013-2015

Tabla 1

Cambios en las dimensiones de la pobreza 2013-2015

\begin{tabular}{lccc}
\hline & 2013 & 2015 & Variación (\%) \\
\hline Carencia por rezago educativo promedio en el & 156 & 139 & -10.90 \\
hogar; & & & \\
Carencia por acceso a los servicios de salud; & 72 & 4 & -94.50 \\
Carencia por acceso a la seguridad social; & 173 & 0 & -100.00 \\
Carencia por calidad y espacios de la vivienda; & 35 & 39 & 11.44 \\
Carencia por acceso a los servicios básicos en la & 137 & 42 & -69.34 \\
vivienda; & & & \\
Carencia por acceso a la alimentación; & 164 & 9 & -94.51 \\
Carencia por ingreso corriente per cápita & & & \\
$\quad$ Ingreso por debajo de la Línea de bienestar & 173 & 170 & $-1,73$ \\
$\quad$ Ingreso por debajo de la Línea de bienestar & 173 & 168 & -2.89 \\
mínimo & & &
\end{tabular}

Nota: Los datos obtenidos en el año 2103 son del Sistema de Focalización del Desarrollo (SIFODE) y los del año 2015 son los recolectados por el cuestionario aplicado.

Fuente: Resultados del análisis estadístico.

\section{DISCUSIÓN}

En esta investigación se analizó el impacto del programa Pensión para Adultos Mayores (PPAM) en las condiciones de bienestar de los hogares beneficiados, mismos que en el año 2013 se encontraban en pobreza extrema en el municipio de Centro, Tabasco. De esta manera, el PPAM logró erradicar la carencia de acceso a la seguridad social al otorgar al $100 \%$ de la población estudiada en un esquema de seguridad social, contribuyendo así a su bienestar mediante el aseguramiento de un ingreso mínimo, así como la entrega de apoyos de protección social a los adultos mayores.

Por otra parte, el Programa del Seguro Popular de Salud, mismo que brinda protección en salud a la población no derechohabiente mediante un seguro de salud, público y voluntario orientado a reducir los gastos médicos de bolsillo y fomentar la atención oportuna a la salud, logró sacar de la carencia por acceso a los servicios de salud al 97\% de las personas investigadas, mismas que en el año 2013 se encontraban carentes de dicha protección. 
IMPACTO DEL PROGRAMA PENSIÓN PARA ADULTOS MAYORES (PPAM) EN TABASCO, MÉXICO. PERÍODO 2013-2015

En lo que respecta a la carencia por rezago educativo, ésta logró disminuirse casi un 11\% lo que representa un logro importante del Instituto de Educación para Adultos de Tabasco, ya que si bien en cifras no constituye un avance tan contundente, toma relevancia toda vez que se trata de adultos mayores de 65 años, mismos que tuvieron la voluntad de estudiar en esta etapa de su vida. Por otro lado, la carencia por acceso a los servicios básicos de la vivienda muestra una disminución sustancial del 69.34\%.

Por su parte, en la carencia por acceso a la alimentación se observó un avance importante ya que se logró una disminución del $94.51 \%$ en los hogares con esta privación. Aquí juegan un papel importante dos programas institucionales: el Programa de Apoyo Alimentario, mismo que otorga hasta $\$ 660.00$ pesos bimestralmente para compra de productos de la canasta básica alimentaria en tiendas Diconsa, así como el Programa de Comedores Comunitarios, el cual busca mejorar las condiciones nutricionales de la población de niños y niñas de 0 a 11 años de edad, mujeres en gestación y lactantes, personas con alguna discapacidad y adultos mayores de 65 años.

Finalmente, los resultados de la investigación permiten afirmar que durante el período 20132015, los hogares con adultos mayores de 65 años en pobreza extrema han mejorado en general sus condiciones de bienestar. Esto se infiere de la disminución observada en sus carencias básicas, exceptuando el indicador de calidad y espacios en la vivienda. Sin embargo, un dato contrastante es que para el año 2015, en el $97.1 \%$ de los hogares encuestados sus ingresos continuaban estando por debajo de la línea de bienestar mínimo, lo que implica que seguían siendo insuficientes para adquirir la canasta básica alimentaria. Ello permite concluir que si bien con la aplicación del PPAM se ha logrado un avance sustancial en la disminución de las carencias sociales de los hogares con adultos mayores, es necesario acompañar este esfuerzo con otros programas institucionales, de manera tal que les permitan mejorar sus ingresos.

La investigación demuestra también que los hogares con adultos mayores están mejorando su nivel de bienestar no como consecuencia de cambios que ocurren de manera natural 0 automática a lo largo del tiempo, si no como resultado de políticas públicas que han logrado mejorar las condiciones socioeconómicas de la población vulnerable. En su conjunto, los resultados sugieren también que los hogares beneficiados del programa Pensión para Adultos 
IMPACTO DEL PROGRAMA PENSIÓN PARA ADULTOS MAYORES (PPAM) EN TABASCO, MÉXICO. PERÍODO 2013-2015

Mayores logran reducir su pobreza a lo largo del tiempo, pero no lo suficiente para que logren escapar de la pobreza, ni para mejorar los ingresos de estas familias.

Al respecto, se ha logrado un gran avance con la estrategia federal denominada Cruzada Nacional contra el Hambre, apoyada con una adecuada focalización de los programas federales, ayudando esto a una reducción de la población en pobreza extrema en el plano nacional. Sin embargo, es recomendable mejorar la coordinación interinstitucional para erradicar de manera eficaz la pobreza en sus diferentes etapas. Asimismo, es recomendable acompañar las políticas públicas con otros programas sociales que permitan a estas familias y a sus generaciones futuras generar ingresos por arriba de la línea de bienestar mínimo. 
IMPACTO DEL PROGRAMA PENSIÓN PARA ADULTOS MAYORES (PPAM) EN TABASCO, MÉXICO. PERÍODO 2013-2015

\section{REFERENCIAS}

Banco Mundial, (2001). Informe sobre el desarrollo mundial 2000/2001: lucha contra la pobreza, Washington: Banco Mundial. Recuperado de http://siteresources.worldbank.org/INTPOVERTY/Resources/WDR/Spoverv.pdf

CONEVAL (2015), Medición de la Pobreza. Glosario, Recuperado de http://www.coneval.gob.mx/Medicion/Paginas/Glosario.aspx

CONEVAL (2015), Medición de la Pobreza, indicadores de carencia social. Recuperado de http://www.coneval.gob.mx/Medicion/Paginas/Medici\%C3\%B3n/Indicadores-de-carenciasocial.asp

Garcia, J. F. (2015). Aproximación al estudio de la pobreza en México: Propuesta de política de estado contra la pobreza (1 $1^{\mathrm{a}} \mathrm{Ed}$.). México: UJAT.

Hernández, R., Fernandez, C. y Baptista P. (2006). Metodología de la investigación. (4ª ed.). México: McGraw Hill Interamericana.

Piketty, T. (2014). El capital en el siglo XXI. México: Fondo de Cultura Económica.

Székely, M. (2005). Pobreza y Desigualdad en México entre 1950 y el 2004. Serie Documentos de Investigación, 24. Secretaría de Desarrollo Social. 